\title{
Reconstruction and numerical modelling of the abdominal wall. Application to hernia surgery
}

\author{
B. Hernández-Gascón 1,2, J. Grasa 1,2, E. Peña 1,2, B. Calvo 1,2 \\ IApplied Mechanics in Bioingineering (AMB) \\ Instituto de Investigación en Ingeniería de Aragón (I3A) \\ Universidad de Zaragoza, Mariano Esquillor s/n, 50018, Zaragoza, Spain. \\ Tel. +34-976762707, Fax+34-976762043, e-mail: belenhg@unizar.es \\ ${ }_{2}$ CIBBER-BBN. Centro de Investigación en Red en Bioingeniería, Biomateriales y Nanomedicina. \\ Spain
}

\begin{abstract}
Routine hernia repair surgery involves the implant of synthetic mesh. However, this procedure may give rise to several problems causing considerable patient disability. With the aim of improving surgical procedures, the healthy and the herniated human abdomen are simulated using finite element (FE) simulations. For that purpose, a reconstruction of the physiological geometry of a human abdomen was created using magnetic resonance images. Besides, following the anatomy of the abdomen, the different muscles and aponeurosis were defined. Furthermore, collagen fibres were included in each muscle layer and their passive anisotropic mechanical contribution was modelled within the framework of hyperelasticity. In the FE simulation of the abdomen, the constraint imposed by the shoulder is applied and an internal pressure of $23 \mathrm{kPa}$ was applied to the interior abdominal wall to reproduce the abdominal load when standing jumping. After generating a hernia in the front of the abdomen, different prostheses (Surgipro ${ }^{\circledR}$, Optilene ${ }^{\circledR}$ and Infinit ${ }^{\circledR}$ ), modelled using a membrane model, are placed in the defect to simulate the behaviour of the abdomen after the surgical procedure. In the healthy abdomen, maximal principal stresses (MPS) and displacements (MD) appear in the front of the belly. On the other hand, once the hernia is created and the mesh is placed, the MD and MPS are higher than those attained in the healthy abdomen. Thus, just after surgery, surgical repair procedure does not fully restore normal physiological conditions and the risk of hernia recurrence by the suture is high due to the stress concentration.
\end{abstract}

